\title{
Picosecond thermometer in the amide I band of myoglobin
}

\author{
Austin, R.H.; Xie, A.; Meer, L. van der; Redlich, B.; Lindgård, Per-Anker; Frauenfelder, H.; Fu, D.
}

Published in:

Physical Review Letters

Link to article, DOI:

10.1103/PhysRevLett.94.128101

Publication date:

2005

Document Version

Publisher's PDF, also known as Version of record

Link back to DTU Orbit

Citation $(A P A)$ :

Austin, R. H., Xie, A., Meer, L. V. D., Redlich, B., Lindgård, P-A., Frauenfelder, H., \& Fu, D. (2005). Picosecond thermometer in the amide I band of myoglobin. Physical Review Letters, 94(12), 128101.

https://doi.org/10.1103/PhysRevLett.94.128101

\section{General rights}

Copyright and moral rights for the publications made accessible in the public portal are retained by the authors and/or other copyright owners and it is a condition of accessing publications that users recognise and abide by the legal requirements associated with these rights.

- Users may download and print one copy of any publication from the public portal for the purpose of private study or research.

- You may not further distribute the material or use it for any profit-making activity or commercial gain

- You may freely distribute the URL identifying the publication in the public portal 


\title{
Picosecond Thermometer in the Amide I Band of Myoglobin
}

\author{
Robert H. Austin, ${ }^{1}$ Aihua Xie, ${ }^{2}$ Lex van der Meer, ${ }^{3}$ Britta Redlich, ${ }^{3}$ Per-Anker Lindgård, ${ }^{4}$ Hans Frauenfelder, ${ }^{5}$ and Dan Fu ${ }^{6}$ \\ ${ }^{1}$ Department of Physics, Princeton University, Princeton, New Jersey 08544, USA \\ ${ }^{2}$ Department of Physics, Oklahoma State University, Stillwater, Oklahoma 74078, USA \\ ${ }^{3}$ FOM Institute for Plasma Physics, Edisonbaan 14, Nieuwegein, The Netherlands \\ ${ }^{4}$ Materials Science Department, Ris $\phi$ National Laboratory, DK-4000 Roskilde, Denmark \\ and QUP Centre, Danish Technical University, DK-2800, Lyngby, Denmark \\ ${ }^{5}$ Theory Division T10, Los Alamos National Laboratory, Los Alamos, New Mexico 87545, USA \\ ${ }^{6}$ Department of Chemistry, Princeton University, Princeton, New Jersey 08544, USA
}

(Received 14 September 2003; revised manuscript received 11 October 2004; published 28 March 2005)

The amide I and II bands in myoglobin show a heterogeneous temperature dependence, with bands at 6.17 and $6.43 \mu \mathrm{m}$ which are more intense at low temperatures. The amide I band temperature dependence is on the long wavelength edge of the band, while the short wavelength side has almost no temperature dependence. We compare concepts of anharmonic solid-state crystal physics and chemical physics for the origins of these bands. We suggest that the long wavelength side is composed of those amino acids which hydrogen bond to the hydration shell of the protein, and that temperature dependent bands can be used to determine the time it takes vibrational energy to flow into the hydration shell. We determine that vibrational energy flow to the hydration shell from the amide I takes approximately 20 ps to occur.

DOI: 10.1103/PhysRevLett.94.128101

PACS numbers: 87.15.Aa

It is well known that the vibrational bands of proteins, including ligands that bind to active groups such as iron, are heterogeneously broadened [1-3]. This heterogeneity can be used to map out the conformational substates of proteins and their exchange on the seconds to days time scale using conventional Fourier-transform spectroscopy, and on the subpicosecond time scale two-dimensional (2D) picosecond infrared measurements can measure the interaction of adjacent amino acids [4,5]. The subpicosecond measurements have typically been done on small oligopetides in order to preserve simplicity in mapping out the matrix of amino acid-amino acid interactions. The amide I band of a typical globular protein such as myoglobin will be more spectrally complex because it has (1) more than one set of secondary structures such as the $\alpha$-helix, (2) a clearly defined outer shell of amino acids in contact with the solvent, and (3) a set of amino acids which are internal and do not hydrogen bind to the solvent. The heterogeneity of the amide I band, in fact, shows itself in the strongly temperature dependent changes in the infrared absorption spectrum [6,7]. In this Letter we use this temperature dependent heterogeneity to probe the picosecond dynamics of vibrational energy flow in myoglobin using relatively narrow, high energy microjoule picosecond IR pulses which probe both the anharmonic response of the amino acids and the time it takes for the system to thermalize within the amide I transitions as opposed to the far-infrared collective modes [8].

We first present the static IR experiments which measure the thermodynamic response of a system to temperature changes. Sperm whale myoglobin (Mb) (Sigma Chemical, St. Louis, MO) was dissolved in a glass-forming $75 \%$ deuterated glycerol $-25 \% \quad \mathrm{D}_{2} \mathrm{O}(\mathrm{v} / \mathrm{v})$ solvent. The final $\mathrm{Mb}$ concentration was $150 \mathrm{mg} / \mathrm{ml}$. The protein solution was held between two $\mathrm{CaF}_{2}$ windows using a $12 \mu \mathrm{m}$ spacer. Optical density at the peak of the amide I band was approximately 1.0 OD units. It is critical in such IR experiments to dissolve the protein in a deuterated solvent because the water $\mathrm{H}_{2}-\mathrm{O}$ scissors mode is also at that wavelength and obscures the amide I band at $6.0 \mu \mathrm{m}$. Note that when a protein like $\mathrm{Mb}$ is placed in a deuterated solvent not all the hydrogens exchange to deuterium: the inner core of the protein does not exchange its hydrogen atoms. This is why there are two amide II bands (the amide II band is due to a "scissoring" $\mathrm{N}-X$ motion [9]) in our sample: a band at $6.50 \mu \mathrm{m}$ due to the $\mathrm{N}-\mathrm{H}$ mode in the inner core of the protein and a band at $6.90 \mu \mathrm{m}$ which is the deuterated N-D modes at the surface of the protein where hydrogen exchange has occurred. The amide I band, which is a $\mathrm{C}=\mathrm{O}$ stretch, is only weakly coupled to the hydrogens. Figure 1 shows the temperature dependence of the IR absorbance (the log base 10 of the transmission) spectrum of sperm whale myoglobin as a function of temperature from 5 to $280 \mathrm{~K}$ in a static gas exchange helium optical cryostat (Oxford Cryogenics OptiStat).

The bands between 2.5 and $5.5 \mu \mathrm{m}$, due to nonexchanged N-H stretching motions, are seen to shift with higher temperature to shorter wavelength, giving rise to a derivative shape to the difference spectra as can easily be seen in Fig. 2(a), which shows the difference spectra of the $\mathrm{Mb}$ absorbance as a function of temperature. However, the bands associated with the protein between 6 and $8 \mu \mathrm{m}$, which are components of the amide I and II bands, do not show shifting of the bands with temperature but instead show the appearance of new bands at low temperatures, at 6.17 and $6.43 \mu \mathrm{m}$ as we show in Fig. 2(b).

Such strong temperature dependent amide I and II absorbance changes are by no means unique to predominantly 

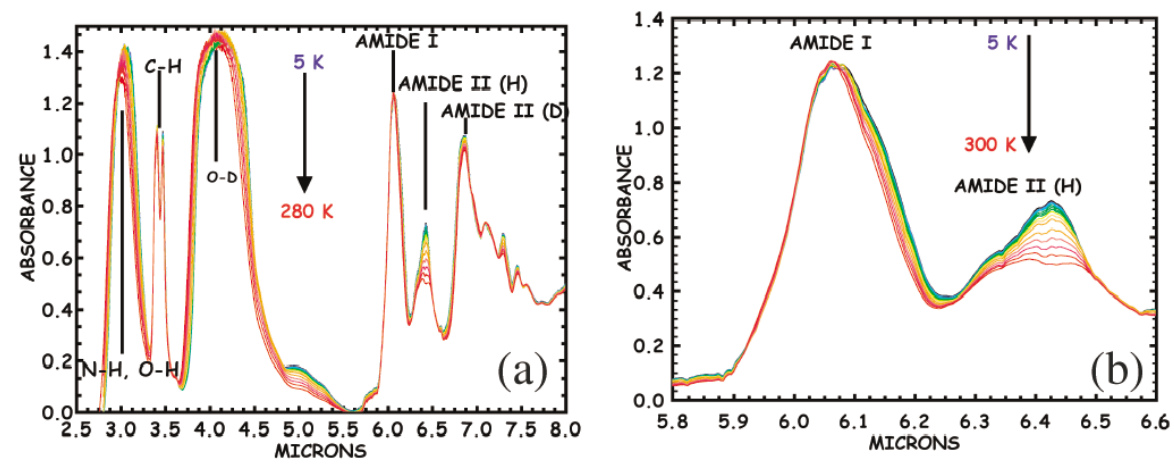

FIG. 1 (color). (a) The infrared absorption spectrum of sperm whale myoglobin as a function of temperature from 5 to $300 \mathrm{~K}$ (blue line to red line). (b) The spectrum from 5.8 to $6.6 \mu \mathrm{m}$.

$\alpha$-helix proteins like $\mathrm{Mb}$; strong temperature dependencies of the amide I and II bands can also be seen in other proteins such as photoactive yellow protein [3] which is a more complex mix of $\alpha$-helix and $\beta$-sheet (data not shown).

We posit two basic models for the origin of the new bands: (1) The amide I and II bands are heterogeneously broadened, amino acids at the surface of the protein hydrogen bond with the hydration shell; this hydrogen bonding is temperature dependent [7]. (2) A dynamical process creates a new state at low temperatures, similar to the exciton trapping of energy in a semiconductor. The dynamical trapping model is the more exotic model [10]. Molecular crystals of the amino acid analog acetanalide show a similar low temperature band [11-13], Alexander and Krumhansl [14] modeled the amide I oscillators of mass $m$ and frequency $\omega_{0}$ embedded in a lattice and coupled anharmonically to the Debye acoustic phonons of the lattice. Relaxation of the excited state of the amide I oscillator due to anharmonic interactions with the local environment (self-trapping) gives rise to a side band which is trapped by a binding energy $\Delta U$. In this model, the temperature dependence of the new band is similar to the low temperature limit of the Debye-Waller factor since phonons carry away the excited vibronic energy and decrease the probability of forming such a self-trapped state [15]. Alexander and Krumhansl calculated that with a Debye spectrum of phonons characterized by a Debye temperature $T_{\mathrm{D}}$ the integrated area $A(T)$ of the self-trapped state would vary with temperature as

$$
A(T) \sim W_{0} \exp \left[-\left(\frac{T}{T_{\mathrm{D}}}\right)^{2}\right],
$$

where $T_{\mathrm{D}}$ is the Debye temperature of the solid, $T_{\mathrm{D}}=\frac{\hbar v}{k_{B}} \times$ $\left(6 \pi^{2} \rho\right)^{1 / 3}$ ( $\rho$ is the number density of the oscillators) [16].

A less exotic model is process (1), where amino acids at the surface of the protein are in contact with bound water molecules, and this interaction increases with decreasing temperature due to the formation of a hydrogen bond. This model certainly explains why all the lines in the IR spectrum change, since hydrogen bonding influences many IR modes, and it explains why the short wavelength side of the amide I band is unaffected by temperature: the buried amino acids at the core of the protein are not in contact with the external solvent. In this model, the hydrogen bond is made with an enthalpic energy difference $\Delta H$ and entropic difference $\Delta S$, and it creates a redshifted population of solvent exposed amino acids. The population of the hydrogen bonded state, presumably measured by its integrated absorbance $A(T)$ then is simply given by

$$
A(T) \sim \frac{1-\tanh \left[-\frac{\Delta H}{k_{B} T}+\frac{\Delta S}{k_{B}}\right]}{2} .
$$

Unfortunately, the fitting of either the phonon model [Eq. (1)] or the conformational splitting model [Eq. (2)] gives equally good fits with reduced $\chi^{2}$ of $\sim 2$, as we show in Fig. 3. Equation (1) does give a good fit to the temperature dependence of the $6.17 \mu \mathrm{m}$ line, and yields a Debye temperature for the amide I backbone delocalized modes of $472 \mathrm{~K}$. Equation (2) can also be fit to the $6.17 \mu \mathrm{m}$ data, and yields a two state splitting of conformational states with $\Delta H / k_{B}=229 \pm 29 \mathrm{~K}$ and $\Delta S / k_{B}=0.36 \pm 0.13$. This is not a highly cooperative structural transition, as can be seen in the large value for the relative width $\Delta T / T_{1 / 2}$ of the struc-
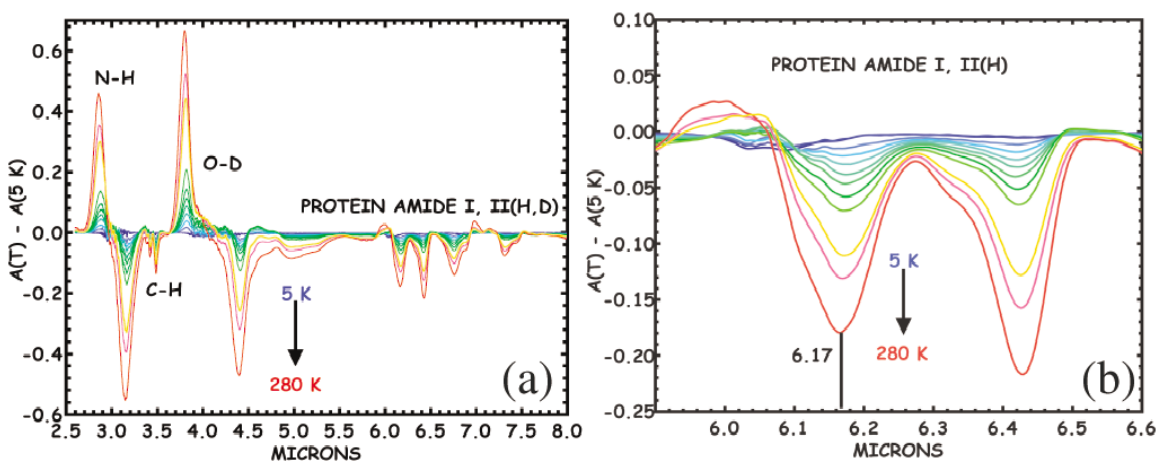

$128101-2$
FIG. 2 (color). (a) The infrared difference absorption spectrum ( $5 \mathrm{~K}$ base) of sperm whale myoglobin as a function of temperature from 10 to $280 \mathrm{~K}$ (blue line to red line), from 2.8 to $8.0 \mu \mathrm{m}$. (b) The difference of the amide I and amide II regions of $\mathrm{Mb}$ ( $5 \mathrm{~K}$ base) as a function of temperature. 


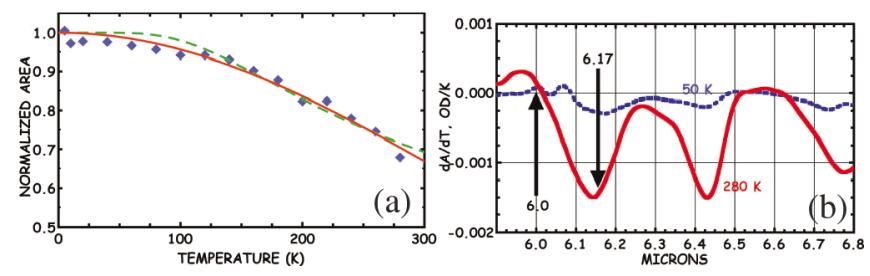

FIG. 3 (color). (a) Fits of the temperature dependence of the IR absorbance of the $6.17 \mu \mathrm{m}$ line in $\mathrm{Mb}$ (solid diamonds). The fit of Eq. (1) to this area is shown as the solid line (red line), and the fit to Eq. (2) is given by the dashed line (green). (b) The temperature derivative $d A / d T$ in $\mathrm{OD} / \mathrm{K}$ of the absorbance at 50 (blue dashed line) and $280 \mathrm{~K}$ (red solid line) between 5.9 and $6.6 \mu \mathrm{m}$.

tural transition. The lesson is that static measurements cannot reveal unambiguously the nature of the IR changes seen.

Now that we have established that $\mathrm{Mb}$ in the amide I and II IR regions has strongly temperature dependent bands at 6.17 and $6.43 \mu \mathrm{m}$, we examine the picosecond IR dynamics of the $6.17 \mu \mathrm{m}$ band. Since this band is sensitive to temperature, we can use it as an indicator of how long it takes a mode-specific pumped energy quantum to flow to the presumably thermodynamic limit seen in the steadystate IR spectra discussed above. In that sense, the $6.17 \mu \mathrm{m}$ band acts as a thermometer, a measure of the thermal temperature of the sample after a picosecond duration input of energy. Figure 3(b) gives the calibration of this "thermometer." The time course of the change tells us at the picosecond time scale the rate at which energy flows into the hydration shell.

The picosecond pump-probe experiments were done at the free electron laser FELIX in Holland [17]. Single micropulses of approximately $1.5 \mathrm{ps}$ duration and $10 \mu \mathrm{J}$ energy were extracted from the $5 \mathrm{~Hz}$ repetition rate macropulse of FELIX. Each trace represents about $10 \mathrm{~min}$ of averaging; a single sample was used for the experiments over the course of a few hours. Since the present experiments use single micropulses and not full macropulses which overheat the sample [18], unlike our previous work which probed long-lived states in $\mathrm{Mb}$ [19], it was possible to do low temperature high intensity pump-probe measurements.

Pulse widths of $1.5 \mathrm{ps}$ were set to achieve $10 \mathrm{~cm}^{-1}$ spectral resolution $(0.040 \mu \mathrm{m}$ at $6 \mu \mathrm{m})$, and in this way the short and long wavelength sides of the band could be examined. Shorter pulses, such as 0.5 ps pulses with $30 \mathrm{~cm}^{-1}$ spectral width, provide information across the band and can be used to determine the anharmonic shift of about $5 \mathrm{~cm}^{-1}$ in the amide I band [19] but compromise spectral selectivity. The pulse was split into a high energy pump pulse of $1 \mu \mathrm{J}$ energy delivered via an optical delay line to the sample and two probe pulses. The measurement probe pulse measured transmission changes due to the pump pulse; the other followed the measurement pulse by $4 \mathrm{~ns}$ and served as a reference pulse used to determine absolute transmission changes. A high speed infrared detector (Boston Electronics) and a digital oscilloscope
(Tektronix TDS 3052) were used to determine transmission changes. The sample was held at atmospheric pressure in a static gas exchange helium flow optical cryostat (Oxford Cryogenics OptiStat). The final focus was done with a $100 \mathrm{~mm}$ focal length off-axis parabolic reflector. Spot sizes were measured to be $50 \mu \mathrm{m}$ FWHM as determined by pinhole energy transmission, resulting in peak intensities of approximately $10^{10} \mathrm{~W} / \mathrm{cm}^{2}$ at the focal spot. We estimate that the final temperature rise in the focal region due to a single $1.0 \mu \mathrm{J}$ IR pulse to be $10 \mathrm{~K}$ at $280 \mathrm{~K}$. Since we know the temperature dependence $d A / d T$ as a function of wavelength for our sample, we can predict that the $280 \mathrm{~K}$ thermalized transmission change of the sample will be $1.5 \times 10^{-2}$ at $\lambda=6.17 \mu \mathrm{m}$ and at least 10 times less than this at $6.0 \mu \mathrm{m}$. At $50 \mathrm{~K}$ we do not know the specific heat accurately but we can guess that it is half that of the $280 \mathrm{~K}$ value [20], and so estimate that the thermalized transmission change will be $0.8 \times 10^{-2}$ at $\lambda=6.17 \mu \mathrm{m}$.

Figure 4 shows the pump-probe response of $\mathrm{Mb}$ at 6.00 and $6.17 \mu \mathrm{m}$ at 50 and $280 \mathrm{~K}$. The main amide I band at the $6.00 \mu \mathrm{m}$ band shows a prompt bleach due to saturation followed by a decay time of 2 ps (the relatively wide pumpprobe width of 1.5 ps strongly convolutes the signal), with decay to the baseline. However, at $6.17 \mu \mathrm{m}$ we see a fast bleaching signal at the pump that recovers followed by a rising transmission change; this long time bleach is presumably due to the heating of the mode responsible for the low temperature band and/or thermalization of the sample.

The rise time of the posttransient picosecond bleach at $50 \mathrm{~K}$ is $28 \pm 4$ ps with an amplitude (transmission change) of $2.5 \times 10^{-2}$, while at $280 \mathrm{~K}$ the rise time was $16 \pm 6 \mathrm{ps}$ with an amplitude of $1.0 \times 10^{-2}$. While the amplitude of the $280 \mathrm{~K}$ signal is roughly what we expected, the expected thermalized transmission change (assuming a $20 \mathrm{~K}$ temperature rise due to the single micropulse) at $50 \mathrm{~K}$ is considerably larger than we expected, indicating that the band is populated to greater than the thermal value at low temperatures. The time constants of the rise time are difficult to interpret at present because of the complexity of using anharmonic energy relaxation in a complex system. Presumably true thermal equilibration occurs via
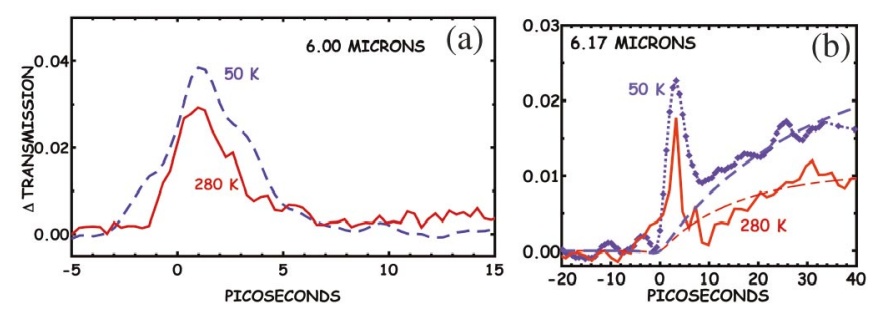

FIG. 4 (color). (a) The pump-probe response at $6.00 \mu \mathrm{m}$ for a $\mathrm{Mb}$ sample. The dashed (blue) line is $50 \mathrm{~K}$, and the solid (red) line is $280 \mathrm{~K}$. (b) The pump-probe response at $6.17 \mu \mathrm{m}$ for a Mb sample. The solid (red) line is the response at $280 \mathrm{~K}$, and the dotdashed (blue) line is the response at $50 \mathrm{~K}$. Fits to the rise in the long time response are shown as a dashed (blue) line for $50 \mathrm{~K}$, and long-short dashed (red) line for $280 \mathrm{~K}$. 


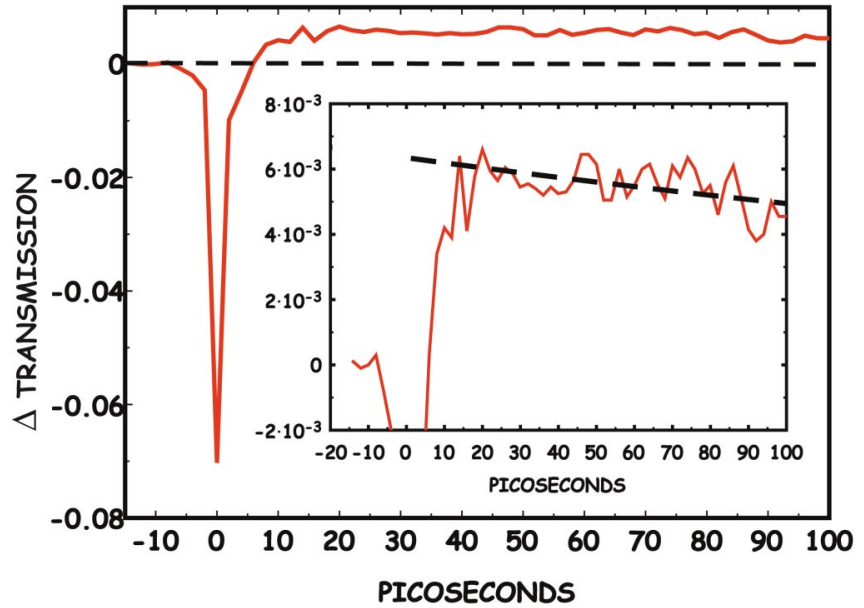

FIG. 5 (color). The pump-probe response at $6.120 \mu \mathrm{m}$ for $\mathrm{Mb}$ at $300 \mathrm{~K}$, long time scan. The inset shows the signal expanded on a vertical scale. The dashed line in the inset is a fit to an exponential decay with time constant $500 \pm 100$ ps.

energy flow through the low frequency $\mathrm{THz}$ collective modes of the system. We have done an earlier $280 \mathrm{~K}$ experiment pumping $\mathrm{THz}$ modes in bacteriorhodopsin at FELIX [8] which observed an unusually long thermalization time of $500 \mathrm{ps}$ of the $\mathrm{THz}$ collective modes. Figure 5 shows the pump-probe signal at $6.120 \mu \mathrm{m}$ at longer times; this experiment was done using an optical parametric oscillator laser at Princeton University after the main experiments were done at FELIX, and the different wavelength and shorter pulse width of the OPO system have changed the sign of the initial signal. Surprisingly, the presumably thermal equilibrium response is not quite flat at long times and, in fact, shows a decay with a time constant of $500 \pm 100 \mathrm{ps}$, the same value observed in the FIR pump-probe experiment. The implications of this relaxation are not clear at present.

Unfortunately we know very little about the response of hydrogen bonded polymers to high intensity picosecond pulses which probe the far anharmonic responses of the system. The anharmonicity parameter for the amide I band for small perturbative fields which result in less than $1 \%$ transmission changes is known to be about $10 \mathrm{~cm}^{-1}$, but in our case we are driving the system quite hard leading to $3 \%$ transmission changes so that we are moving into large values of the anharmonic response and a possibly longlived deformation of the lattice. Before we can claim to understand the anharmonic response of a protein to single mode pumping of large amounts of energy, we need to explore much more of this space because it is related to the anharmonic dynamics of conformational motion [21]. We know that the Debye-Waller factors in a protein are highly dependent on the position of the amino acids [22], indicating high flexibility at the hydration shell and much more restricted motions in the interior of the protein. There already is information from a time-resolved $\mathrm{x}$ ray that significant atomic motion can occur at 150 ps [23], but much is to be learned about the coherent dynamics on the picosecond time scale, and the approach to thermal equilibrium at longer time scales approaching nanoseconds.

This work was supported by the Office of Naval Research and the Stichting voor Fundamenteel Onderzoek der Materie (FOM).

[1] G. U. Nienhaus, J. R. Mourant, and H. Frauenfelder, Proc. Natl. Acad. Sci. U.S.A. 89, 2902 (1992).

[2] G. U. Nienhaus, J.D. Muller, B.H. McMahon, and H. Frauenfelder, Physica (Amsterdam) 107D, 297 (1997).

[3] A. H. Xie, W. D. Hoff, A. R. Kroon, and K. J. Hellingwerf, Biochemistry 35, 14671 (1996).

[4] P. Hamm, M. H. Lim, and R. M. Hochstrasser, J. Phys. Chem. B 102, 6123 (1998).

[5] J. Edler and P. Hamm, J. Chem. Phys. 117, 2415 (2002).

[6] F. Demmel, W. Doster, W. Petry, and A. Schulte, Eur. Biophys. J. Biophys. Lett. 26, 327 (1997).

[7] E. S. Manas, Z. Getahun, W. W. Wright, W. F. DeGrado, and J. M. Vanderkooi, J. Am. Chem. Soc. 122, 9883 (2000).

[8] A. Xie, L. van der Meer, and R. H. Austin, Phys. Rev. Lett. 88, 018102 (2002).

[9] Infrared Spectroscopy of Biomolecules, edited by H. H. Mantsch and D. Chapman (Wiley, New York, 1996).

[10] Per-Anker Lindgård and A. M. Stoneham, J. Phys. Condens. Matter 15, V5 (2003).

[11] G. Careri, U. Buontempo, F. Carta, E. Gratton, and A. C. Scott, Phys. Rev. Lett. 51, 304 (1983).

[12] G. B. Blanchet and C. R. Fincher, Phys. Rev. Lett. 54, 1310 (1985).

[13] A. Spire, M. Barthes, H. Kellouai, and G. De Nunzio, Physica (Amsterdam) 137D, 392 (2000).

[14] D. M. Alexander and J. A Krumhansl, Phys. Rev. B 33, 7172 (1986).

[15] S. B. Tripathi, J.D. Pandey, N.D. Tripathi, and S. K. Pandey, Acustica 35, 278 (1976).

[16] D. Mermin, J. Math. Phys. (N.Y.) 7, 1038 (1966).

[17] A.F.G. van der Meer and M.J. van der Wiel, Appl. Spectrosc. 51, 574 (1997).

[18] R. H. Austin and A. Xie, Nucl. Instrum. Methods Phys. Res., Sect. A 407, 504 (1998).

[19] A. Xie, A. F. G. van der Meer, W. Hoff, and R. H. Austin, Phys. Rev. Lett. 84, 5435 (2000).

[20] C. Talon, Q.W. Zou, M.A. Ramos, R. Villar, and S. Vieira, Phys. Rev. B 65, 012203 (2002).

[21] H. Frauenfelder, B. H. McMahon, R. H. Austin, K. Chu, and J. T. Groves, Proc. Natl. Acad. Sci. U.S.A. 98, 2370 (2001).

[22] H. Frauenfelder, G. A. Petsko, and D. Tsernoglou, Nature (London) 280, 558 (1979).

[23] F. Schotte, M. Lim, T. A. Jackson, A. V. Smirnov, J. Soman, J. S. Olson, G. N. Phillips, M. Wulff, and P. A. Anfinrud, Science 300, 1944 (2003). 